\title{
A Study of Fluid Interface Configurations in Exploration Vehicle Propellant Tanks
}

\author{
Gregory A. Zimmerli ${ }^{1}$ \\ NASA Glenn Research Center, Cleveland, OH 44135 \\ Marius Asipauskas ${ }^{2}$ \\ National Center for Space Exploration Research, Cleveland, $\mathrm{OH} 44135$ \\ and \\ Yongkang Chen $^{3}$, Mark M. Weislogel ${ }^{4}$ \\ Portland State University, Portland, OR 97201-0751
}

\begin{abstract}
The equilibrium shape and location of fluid interfaces in spacecraft propellant tanks while in low-gravity is of interest to system designers, but can be challenging to predict. The propellant position can affect many aspects of the spacecraft such as the spacecraft center of mass, response to thruster firing due to sloshing, liquid acquisition, propellant mass gauging, and thermal control systems. We use Surface Evolver, a fluid interface energy minimizing algorithm, to investigate theoretical equilibrium liquid-vapor interfaces for spacecraft propellant tanks similar to those that have been considered for NASA's new class of Exploration vehicles. The choice of tank design parameters we consider are derived from the NASA Exploration Systems Architecture Study report. The local acceleration vector employed in the computations is determined by estimating low-Earth orbit (LEO) atmospheric drag effects and centrifugal forces due to a fixed spacecraft orientation with respect to the Earth or Moon, and rotisserie-type spacecraft rotation. Propellant/vapor interface positions are computed for the Earth Departure Stage and Altair lunar lander descent and ascent stage tanks for propellant loads applicable to LEO and low-lunar orbit. In some of the cases investigated the vapor ullage bubble is located at the drain end of the tank, where propellant management device hardware is often located.
\end{abstract}

\section{Nomenclature}

a $\quad=$ acceleration

$A \quad=$ area

Bo $\quad=$ Bond number

$C \quad=$ center of mass distance vector

$\mathrm{CM}=$ center of mass location

$C_{\mathrm{D}} \quad=$ drag coefficient

$E \quad=$ potential energy

$F \quad=$ force

$1, \mathrm{~s}, \mathrm{v}=$ subscripts indicating liquid, solid, and vapor

$r \quad=$ generic spatial coordinate

$R \quad=$ tank radius

$S \quad$ = surface area

$T \quad=$ spacecraft orbital period

\footnotetext{
${ }^{1}$ Aerospace Engineer, 21000 Brookpark Road, MS 301-3.

${ }^{2}$ Research Associate, 21000 Brookpark Road, MS 110-3.

${ }^{3}$ Research Associate, Mechanical and Materials Engineering, P O Box 751-ME, Senior Member AIAA.

${ }^{4}$ Professor, Mechanical and Materials Engineering, P O Box 751-ME, Senior Member AIAA. 
$\begin{array}{ll}v & =\text { spacecraft velocity } \\ V & =\text { volume } \\ \rho & =\text { fluid density } \\ \sigma & =\text { surface tension } \\ \varphi & =\text { acceleration vector angle } \\ \psi & =\text { angle between acceleration vector and center of mass vector }\end{array}$

\section{Introduction}

$\mathrm{I}_{\mathrm{r}}^{\mathrm{n}}$ 2005 NASA released an Exploration Systems Architecture Study (ESAS) report ${ }^{1}$ that provided baseline recommendations for a new class of space exploration and launch vehicles. These vehicles require the use of highperformance liquid propellants for the propulsion systems, and the propellants account for approximately $75 \%$ of the overall system mass after reaching low-Earth orbit (LEO). Propellant management is a critical consideration in the system design. Efficient and wise management of the propellants affects the spacecraft design, performance, and even spacecraft stability. The design of sub-systems for thermal management, liquid acquisition, and propellant quantity gauging are important considerations in the design cycle, and successful implementation of these subsystems relies on accurate knowledge of fluid position and properties. The thermophysical properties of the propellants are generally well known, but the static spatial distribution of the fluid in the propellant tanks in microgravity is generally regarded as uncertain.

Experiments and calculations to investigate the liquid-vapor interface configurations in low-gravity propellant tanks dates back to the early days of the space program. Much of the early work was summarized by Hastings et al. in a NASA contractor report ${ }^{2}$. Calculations of the minimal surface energy interface were limited to simple problems, but certainly aided in understanding what shape the surface might take in low gravity. Calculations of the surface shape remained rather difficult until the early 1990's, when K. Brakke released a computer program to calculate the minimal energy of a surface, given a set of constraints or boundary conditions ${ }^{3}$. The program, known as Surface Evolver (SE), has since been used to successfully predict liquid-vapor interface shapes in microgravity ${ }^{4-6}$, and has also been applied to gauging the amount of propellant in satellites ${ }^{7}$, and modeling of propellant management devices $^{8}$. Here, we use the SE code to investigate possible liquid-vapor interface configurations in the next class of NASA exploration vehicle tanks using realistic boundary conditions. The examples chosen illustrate the types of problems that can be solved, and are certainly not meant to be the only possibilities. Several fluid interface configurations are calculated for the Earth Departure Stage and Altair vehicle tanks using the SE code with inputs based on the thermophysical properties of the fluids, tank and spacecraft design parameters from the ESAS report, and calculation of the forces acting on the spacecraft.

In Section II the physical model used for the spacecraft is presented. Section III discusses the forces on the spacecraft that were used as inputs to the SE model. A description of the boundary conditions used in the SE code is given in Section IV, and the results of the calculations are presented in Section V as graphical representations of the liquid-vapor interface in the tank.

\section{Spacecraft model}

The model chosen for this study is the baseline recommended spacecraft model in the ESAS report referred to as the 1.5-Launch, LV 27.3 configuration. Briefly, in this configuration the Earth Departure Stage (EDS) and the Lunar Surface Access Module (LSAM, now named the Altair vehicle) are launched together on an Ares-V rocket and rendezvous in LEO with the Crew Exploration Vehicle (CEV). The EDS is used as an upper stage for the Ares-V, and thus is only partially filled with propellant in LEO. After performing a trans-lunar injection burn, the EDS is discarded leaving only the Altair and CEV on a coast to the moon. The Altair descent stage performs the lunar orbit injection burn, and the two vehicles orbit the moon before undocking and lunar descent of the Altair vehicle.

A schematic of the model adopted here for the EDS, Altair, CEV vehicle stack is shown in Figure 1. Dimensional lengths of the spacecraft and propellant tanks used in our model were estimated from information and figures in the ESAS report. The values used here are not reflective of a current vehicle design, they are simply estimates based on one of the ESAS concepts. The goal of this work is to show that given a specific vehicle configuration and boundary conditions, the equilibrium fluid position in the propellant tanks can often be readily calculated.

The propellant tank volumes in our model are based on the ESAS estimates for the required propellant mass for each vehicle, and we further assumed that the propellants were loaded into the tanks at their normal boiling points and that the initial volume fill level is $98 \%$ for the EDS liquid oxygen (LOX) and liquid hydrogen (LH2) tanks (1 each), $95 \%$ for the Altair descent stage LOX (2 each) and LH2 tanks (6 each), and $90 \%$ for the Altair ascent stage 
LOX and liquid methane (LCH4) tanks (2 each). We assumed oxidizer/fuel mass mixture ratios of $6: 1$ for the LOX/LH2 tanks, and 3.6:1 for LOX/LCH4. The EDS LOX and LH2 tank diameters were both chosen to be $26.5 \mathrm{ft}$, slightly less than the ESAS $27.5 \mathrm{ft}$ vehicle diameter. In the SE tank model, the EDS and Altair descent stage tanks are assigned a diameter, cylinder section length $L$, capped by ellipsoidal domes of height $h$. The Altair ascent stage tanks are modeled as spheres. Table 1 summarizes the EDS and Altair tank dimensions used in our model. The CEV Service Module propellant tanks were not considered in this study.

The center of mass of a vehicle stack was calculated using a lumped element one-dimensional model for each vehicle. An appropriate propellant mass and location was added to the one-dimensional vehicle dry-mass model to calculate the spacecraft center of mass. In LEO, the EDS tanks were assigned a fill level of $45 \%$, and in low-lunar orbit (LLO) the Altair descent stage tanks (-D) were assigned a fill level of $41 \%$. Both values are based on information extracted from the ESAS report.

Figure 1 indicates two locations for the vehicle center of mass (CM), based on two different stack configurations. CM-1 is the calculated center of mass of the combined Altair-EDS vehicle stack with propellant in LEO, before docking with the CEV. CM-2 is the calculated center of mass of the Altair-CEV vehicle stack with propellant in LLO.

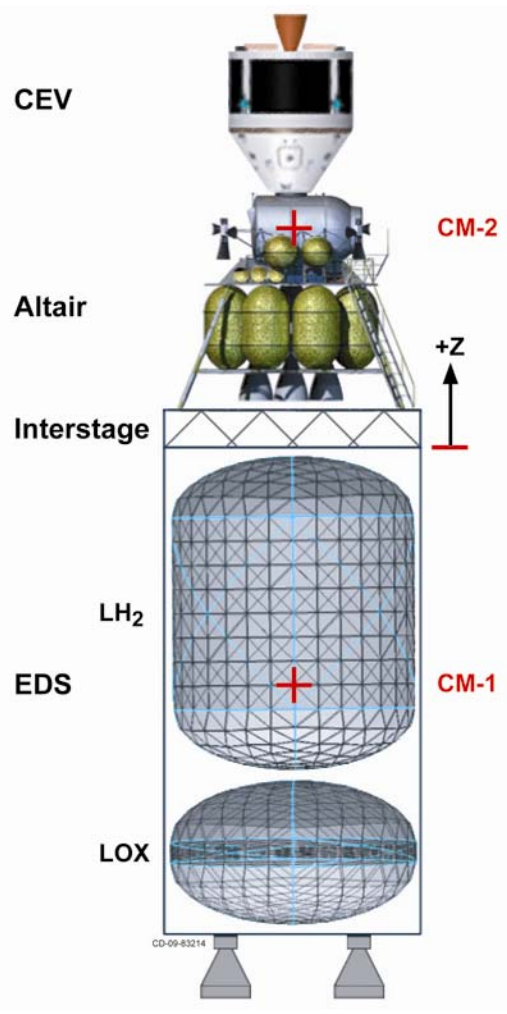

Figure 1. Schematic of the spacecraft model used in this study.

\begin{tabular}{ccccc}
\multicolumn{6}{c}{ Table 1. Tank dimensions and volume. } \\
\hline \hline Vehicle, Tank & Radius $(\mathrm{m})$ & $\begin{array}{c}\text { Cylinder } \\
\text { L }(\mathrm{m})\end{array}$ & Dome height $h(\mathrm{~m})$ & Vol. $\left(\mathrm{m}^{3}\right)$ \\
\hline EDS, LOX & 4.038 & 0.683 & 2.019 & 172.9 \\
EDS, LH2 & 4.038 & 6.373 & 2.019 & 464.4 \\
Altair-D, LH2 & 1.000 & 2.160 & 0.500 & 8.880 \\
Altair-D, LOX & 1.060 & 2.150 & 0.500 & 9.943 \\
Altair-A, LOX & 0.754 & 0.000 & 0.754 & 1.796 \\
Altair-A, LCH4 & 0.685 & 0.000 & 0.685 & 1.346 \\
\hline \hline
\end{tabular}

Table 2. Spacecraft mass distribution model in LEO.

\begin{tabular}{ccc}
\hline \hline Vehicle/Tank & $m(\mathrm{~kg})$ & location, $z(\mathrm{~m})$ \\
\hline CEV, Service Module & 12154 & 13.7 \\
CEV, Crew Module & 8446 & 11.3 \\
Altair, ascent stage dry mass & 6094 & 9.47 \\
Altair, ascent stage propellant & 4715 & 8.53 \\
Altair, descent stage dry mass & 9950 & 5.54 \\
Altair, descent stage propellant & 25105 & 5.46 \\
EDS, liquid hydrogen & 14635 & -3.3 \\
EDS dry mass & 20012 & -11.7 \\
EDS, liquid oxygen & 87810 & -15.5 \\
\hline \hline
\end{tabular}

\section{Forces in microgravity}

In the absence of any thrust produced by the spacecraft, the dominant forces acting on the spacecraft will be centrifugal forces due to spacecraft rotation, and atmospheric drag, which will only be present in LEO. Two rotational modes are considered; a rotation about the spacecraft center of mass at the same frequency as the orbital frequency, and a rotisserie-type rotation about the long axis of the spacecraft. The effect of self-gravitation is shown to be comparatively small but may be non-negligible during a trans-lunar coast if spacecraft rotation is absent.

The drag force $F_{D}$ imparted by the thin atmosphere in LEO was estimated using the standard drag force equation, 


$$
F_{D}=\frac{1}{2} \rho v^{2} C_{D} S
$$

where $\rho$ is the density of the atmosphere, $v$ is the spacecraft velocity, $C_{D}$ is the drag coefficient, and $S$ is the spacecraft cross-section area in the direction of motion, or shadow area. The LEO orbital velocity at an altitude of $300 \mathrm{~km}$ is readily calculated to be $7.7 \mathrm{~km} / \mathrm{s}$, and $S$ is estimated at $S=220 \mathrm{~m}^{2}$ for the EDS/Altair vehicle (without the CEV docked) orbiting in a gravity-gradient orientation where the aft section of the vehicle is pointed to Earth. A value of $C_{D}=2.3$ was used for the drag coefficient, which is a mid-range value for spacecraft of various geometries ${ }^{9}$. The density of the atmosphere in LEO is highly variable depending on solar activity, and we chose an intermediate thermosphere temperature of $980 \mathrm{~K}$ at $300 \mathrm{~km}$, with a corresponding density of $\rho=2.3 \times 10^{-11} \mathrm{~kg} / \mathrm{m}^{3}$ [10]. Using the parameters stated above, the atmospheric drag causes the spacecraft of mass $m_{s}$ to decelerate an amount $a=F_{D} / m_{s}=$ $2.3 \times 10^{-7} \mathrm{~g}$, where $g=9.8 \mathrm{~m} / \mathrm{s}^{2}$ and the spacecraft mass is taken as $m_{s}=152 \mathrm{mT}$. The drag force is in a direction opposite the velocity vector and causes the fluid to move forward in the tanks, in the direction of motion.

Next we consider centrifugal forces due to spacecraft rotation. If the orientation of the spacecraft with respect to the surface of the Earth is fixed, as in a gravity-gradient orientation, for example, then in an inertial reference frame the spacecraft is rotating about a principal axis passing through its center of mass once per orbit. The fluid thus experiences a centrifugal acceleration $a_{c}$ given by

$$
a_{C}=\left(\frac{2 \pi}{T}\right)^{2}\left(r-r_{0}\right)
$$

where $T$ is the period of rotation and $r-r_{0}$ is the distance from the fluid to the axis of rotation of the spacecraft. For a rotational period of 90 minutes, the centrifugal acceleration at $r-r_{0}=10 \mathrm{~m}$ is approximately $1.4 \times 10^{-6} \mathrm{~g}$. In this paper we neglect the gradient in the centrifugal acceleration field that would occur in a real propellant tank due to the $r$ dependence in Eq. (2), and instead simply calculate the centrifugal acceleration at a characteristic liquid-vapor interface location.

To calculate the centrifugal accelerations, we used a simple one-dimensional model for the spacecraft dry mass and propellant mass components in order to calculate the spacecraft center of mass. Table 2 shows the values used in our model, which were derived from the ESAS report and the model shown in Figure 1. For purposes of calculating the center of mass, the dry mass for each vehicle was modeled as a lumped element in the center of the vehicle. The propellant mass was assumed to be settled away from the center of rotation of the vehicle. We considered the centrifugal acceleration on the EDS/Altair vehicles in LEO, and of the Altair/CEV vehicles in LLO. The centrifugal accelerations for the Altair/CEV vehicle tanks in LLO were calculated by assuming the spacecraft had a fixed orientation with respect to the moon's surface, an orbital period of 118 minutes, and a propellant fill level of $41 \%$ for the Altair lander tanks. Figure 1 shows the position of the calculated center of mass of the EDS/Altair vehicle in LEO (CEV not docked), labeled CM-1, and the Altair/CEV vehicle in LLO, labeled CM-2. A $z=0$ reference plane is taken at the interface of the EDS and interstage structure. In our model, the $168 \mathrm{mT}$ EDS/Altair center of mass in LEO is located at CM-1 $=-8.0 \mathrm{~m}$, and the $52 \mathrm{mT}$ Altair/CEV center of mass in LLO is located at CM- $2=+7.5 \mathrm{~m}$. Adding the CEV to our model of the EDS/Altair vehicle in LEO would move the center of mass to $z=-5.6 \mathrm{~m}$, close to the center of the EDS LH2 tank.

In addition to a centrifugal acceleration resulting from a fixed spacecraft orientation with respect to the Earth or Moon, we also consider the effect of a rotisserie-type rotation about the long axis of the spacecraft. The resulting centrifugal acceleration is again given by Eq. (2), and in our model we used a 20 minute rotation period. Such a rotation might be considered in order to achieve a more uniform exposure of the spacecraft to the sun and deep space. The effect of the rotisserie-type rotation was only considered for the off-axis Altair vehicle tanks in LEO, and the atmospheric drag was neglected in these cases.

The effect of self-gravitation was considered, but is found to be small in comparison with the other forces under consideration. To estimate the self-gravitation contribution, one can consider the EDS LOX tank alone, since it is the most massive and dense element on the spacecraft. Approximating the EDS liquid oxygen mass $m_{\mathrm{LOX}}$ as a sphere of radius $r_{L O X}=\left(3 m_{L O X} / 4 \pi \rho\right)^{1 / 3}$ we find that a fluid element on the surface of the sphere would experience a selfgravitational field of $8.6 \times 10^{-8} \mathrm{~g}$, which is more than $10 \times$ smaller than the centrifugal acceleration considered here for the EDS LOX tank. 
Table 3. Summary of propellant tanks and acceleration fields modeled with Surface Evolver.

\begin{tabular}{cccccccc}
\hline Case \# & Tank & Orbit & Volume fill & $a_{y}, a_{z}\left(10^{-6} \mathrm{~g}\right)$ & $|a|\left(10^{-6} \mathrm{~g}\right)$ & $\phi$ (degrees) & Bond number \\
\hline 1 & EDS, LOX & LEO & $45 \%$ & $-0.23,-1.0$ & 1.03 & 13 & 14.13 \\
2 & EDS, LH2 & LEO & $45 \%$ & $-0.23,0.3$ & 0.38 & 143 & 2.13 \\
3 & Altair-D, LH2 & LEO & $95 \%$ & $-0.23,2.0$ & 2.01 & 173 & 0.70 \\
4 & Altair-D, LOX & LEO & $95 \%$ & $-0.23,2.0$ & 2.01 & 173 & 1.91 \\
5 & Altair-A, LOX & LEO & $90 \%$ & $-0.23,2.3$ & 2.31 & 174 & 1.11 \\
6 & Altair-A, LCH4 & LEO & $90 \%$ & $-0.23,2.3$ & 2.31 & 174 & 0.34 \\
7 & Altair-D, LH2 & LLO & $41 \%$ & $-0.21,-0.32$ & 0.38 & 33 & 0.13 \\
8 & Altair-D, LOX & LLO & $41 \%$ & $-0.21,-0.32$ & 0.38 & 33 & 0.36 \\
9 & Altair-A, LOX & LLO & $90 \%$ & $-0.12,-0.04$ & 0.13 & 72 & 0.06 \\
10 & Altair-A, LCH4 & LLO & $90 \%$ & $-0.12,-0.04$ & 0.13 & 72 & 0.02 \\
11 & Altair-D, LH2 & LEO & $95 \%$ & $5.9,2.0$ & 6.23 & 251 & 2.15 \\
12 & Altair-D, LOX & LEO & $95 \%$ & $5.9,2.0$ & 6.23 & 251 & 5.91 \\
13 & Altair-A, LOX & LEO & $90 \%$ & $4.2,2.0$ & 4.79 & 241 & 2.30 \\
14 & Altair-A, LCH4 & LEO & $90 \%$ & $4.2,2.0$ & 4.79 & 241 & 0.70 \\
\hline \hline
\end{tabular}

Table 3 summarizes the spacecraft propellant tank fill-levels and acceleration field components $a_{y}$ and $a_{z}$ used in our analysis of the liquid-vapor interface location. For notational simplicity the acceleration component resulting from a rotisserie-type rotation is labeled $a_{y}$ although it is directed radially outward. The atmospheric drag contribution is also denoted $a_{y}$ and the centrifugal contribution at the orbital period is denoted $a_{z}$. When two forces were considered for the same tank, the orthogonal fields were vector summed and entered into SE as an acceleration field of appropriate magnitude $a$ and direction $\phi$ relative to the local vertical as shown in Figure 2. Table 3 also indicates the Bond number for each case, defined as

$$
B o=\frac{\Delta \rho a R^{2}}{\sigma}
$$

where $\Delta \rho$ is the density difference between the liquid and vapor, $\sigma$ is the liquid-vapor surface tension, and $R$ is the radius of the tank. The Bond number is a dimensionless measure of the ratio of inertial and surface tension forces. Values for the density and surface tension at the normal boiling point were obtained using REFPROP ${ }^{11}$.

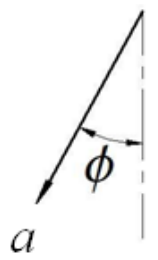

Figure 2. Definition of the acceleration field direction.

\section{Surface Evolver simulation formulation and setup}

As discussed in the introduction, SE has been used by some in the aerospace community for predicting fluid configurations in propellant tanks and other containers. The code has been validated by experiments ${ }^{12,13}$. 
SE finds the minimal potential energy of the system by an energy descent method. In general the potential energy includes gravitational and surface energies, which can be written as

$$
E=\sum_{i} \rho_{i} V_{i} \vec{a} \cdot \vec{C}_{i}+\sum_{j} \sigma_{j} A_{j}
$$

where $\vec{a}$ is the acceleration vector, $V$ is the volume of vapor or liquid, $\vec{C}$ is the distance vector from the center of mass of the liquid or vapor to the origin of the coordinate system (taken at the center of the tank), and $A$ is the area of interface surfaces. The summations in Eq. (4) are over bodies of vapor and liquid and interface surfaces. In this study, interface configurations inside an enclosure (a propellant tank) are investigated. Consequently the total energy for this type of geometry can be reduced to

$$
E_{0}=\Delta \rho V_{\mathrm{v}} \vec{a} \cdot \overrightarrow{C_{\mathrm{v}}}+\sigma_{\mathrm{lv}}\left(A_{\mathrm{lv}}+A_{\mathrm{sv}}\right)
$$

where $\Delta \rho$ is the density difference between the liquid and vapor, and the subscripts denote $\mathrm{v}$ vapor, 1 liquid, and $\mathrm{s}$ solid. The constant energy terms are omitted in Eq. (5) as only the non-constant terms are of interest in the simulation. In addition, Eq. (5) is valid only for perfect wetting fluids. The equation can be further generalized by dividing all the terms on the two sides of the equation by $\sigma_{\mathrm{lv}}$ to obtain

$$
E_{1} \equiv \frac{E_{0}}{\sigma_{\mathrm{lv}}}=\mathrm{Bo}_{1} V_{\mathrm{v}}\left|\overrightarrow{C_{\mathrm{v}}}\right| \cos \psi+\left(A_{\mathrm{lv}}+A_{\mathrm{sv}}\right)
$$

where $\mathrm{Bo}_{1}=\Delta \rho|\vec{a}| / \sigma_{\mathrm{lv}}$ is the unit Bond number and $\psi$ is the angle between $\vec{a}$ and $\overrightarrow{C_{\mathrm{v}}}$. $E_{1}$ is the quantity minimized in our SE simulations.

In the SE computations conducted for this study, the initial condition is an ullage bubble located in the center of the container, as shown in Figure 3. It provides some flexibility as the final position of the interface is dependent on the direction of the acceleration field. This approach is referred to as the Ullage Bubble Method and has been used previously by Collicott ${ }^{8}$. It is straightforward as compared with another approach in SE, the Contact Line Integral Method, in which integrals along the contact lines are included to take into account the interfacial energies between the liquid and the solid surfaces and the volume content of constraints such that the fluid/solid interfaces are not simulated. The ullage bubble method adopted here is especially suitable for perfectly wetting fluids when there are fluid/solid interfaces, such as the cases investigated here.

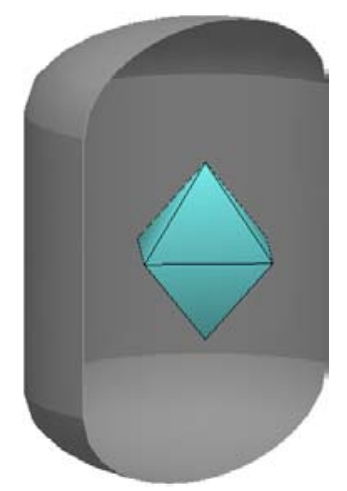

Figure 3. Initial liquid/vapor interface configuration using the Ullage Bubble Method. Only half of the container wall is displayed.

\section{Computation results}

In total there are 14 cases simulated in this study, corresponding to those listed in Table 3. The tank geometry, unit Bond number, and the direction of the acceleration field are the inputs to each SE simulation. The resulting interface configurations, or ullage bubble shapes, are shown in Figures 4-7 below. The meshed surface represents the liquid/vapor interface enclosing the ullage bubble, and the remainder of the tank is occupied by liquid propellant. 
All results presented are computed with a mesh of approximately 4000 vertices. In addition to the interface shape, useful information such as the center of mass of the liquid can also be extracted from the SE computations. The figures indicate the center of the tank and the center of mass of the liquid. A vector within a circle is included as well to indicate the direction of the net acceleration.

Figure 4 shows the liquid-vapor interfaces in the Earth Departure Stage LOX and LH2 tanks in LEO. In this model, the ullage bubble is located at the "top" of the LOX tank (the end opposite the engine feedline drain), but is located at the bottom (drain) end of the LH2 tank. Any consideration to locate propellant management device hardware at the drain end of the tank should account for the possibility that there may not be any liquid at that location while in LEO. The interface configuration in the EDS LH2 tank would be significantly different if the CEV were docked to the Altair vehicle, as this would move the center of mass of the spacecraft close to the center of the LH2 tank. From Figure 4 one can also see that the center of mass of the liquid oxygen, which accounts for over half of the spacecraft mass in this model, is predicted to be shifted off-axis by approximately $2.7 \mathrm{ft}$. Such information is likely to be of interest to spacecraft system engineers, and shows the utility of performing these types of simulations.

The interface configurations for the Altair vehicle tanks in LEO are shown in Figure 5, and are based on an acceleration field resulting from a fixed Earth-spacecraft orientation plus orbital drag. A notable feature, as expected based on the acceleration vector, is that the ullage bubble is located at the bottom of the tank. Figure 6 shows the same tanks where the acceleration field is the result of a fixed Earth-spacecraft $\mathrm{z}$-axis orientation plus a 20 minute period rotisserie-type rotation about the z-axis (orbital drag was neglected).

Interface configurations for the Altair vehicle tanks in LLO are shown in Figure 7, where the acceleration field is the result of a fixed moon-spacecraft orientation. For these simulations, the principal axis of rotation is taken as a horizontal line passing through CM-2 in Figure 1. The spacecraft rotates about this axis once per orbit, leading to the centrifugal accelerations shown in Table 3. The descent stage tanks have been partially depleted of propellant as a result of the lunar orbit injection burn. As can be seen from Figure 7, the liquid covers the drain end of the ascent and descent stage tanks in this model.
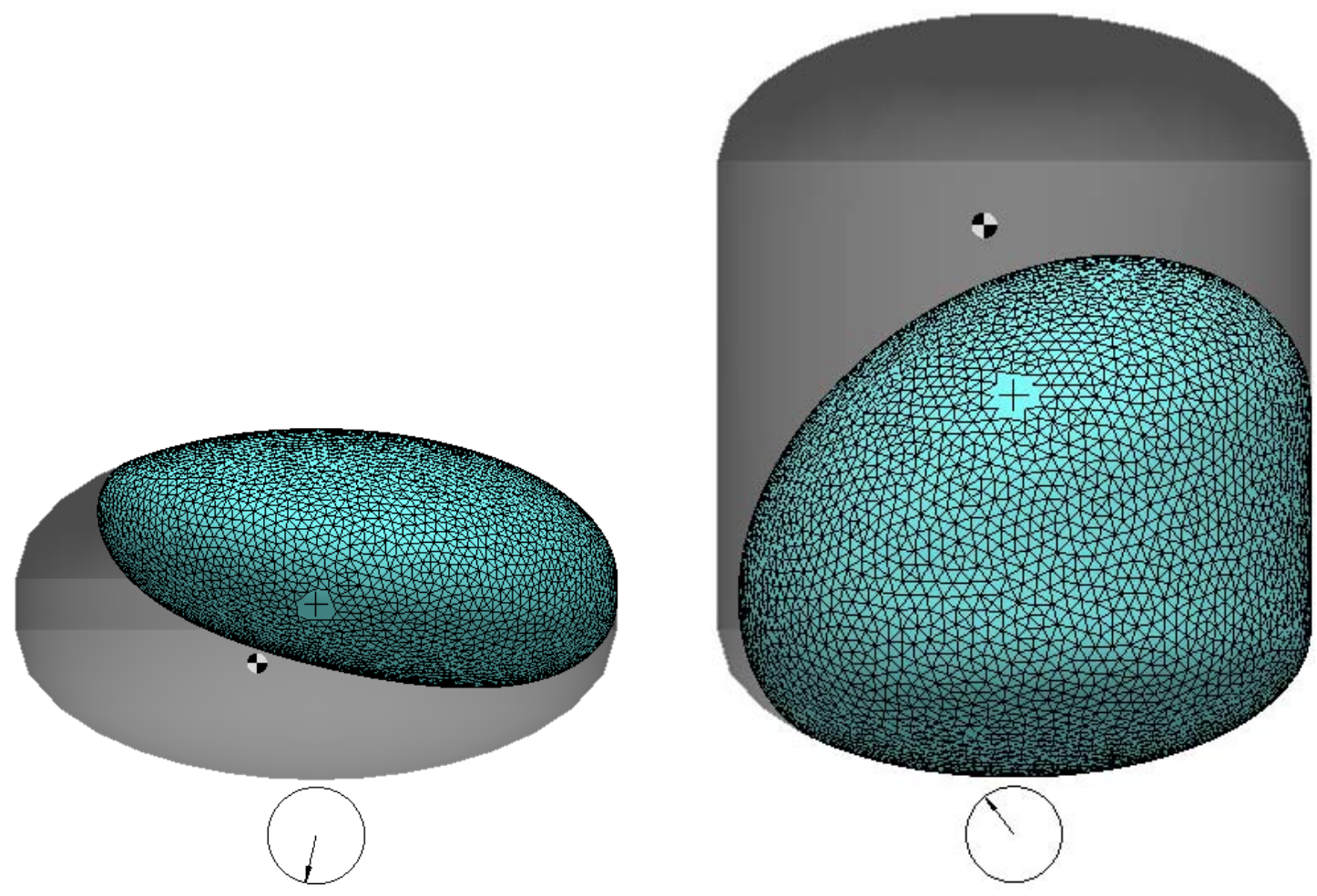

Figure 4. Ullage bubble shapes for the EDS LOX tank (left, Case 1) and LH2 tank (right, Case 2) in LEO. The acceleration field is the result of a fixed Earth-spacecraft orientation plus orbital drag. 


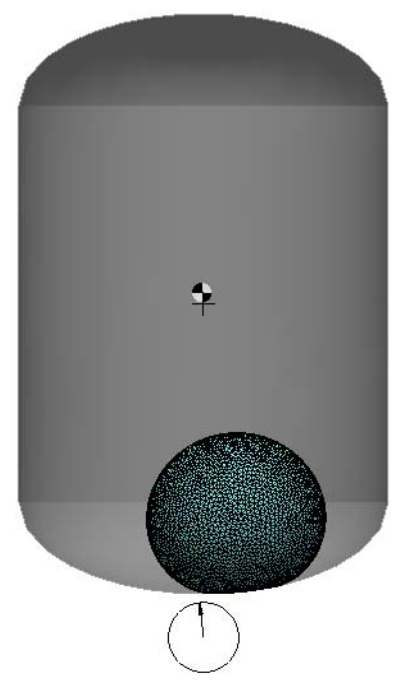

Case 3

Descent LH2

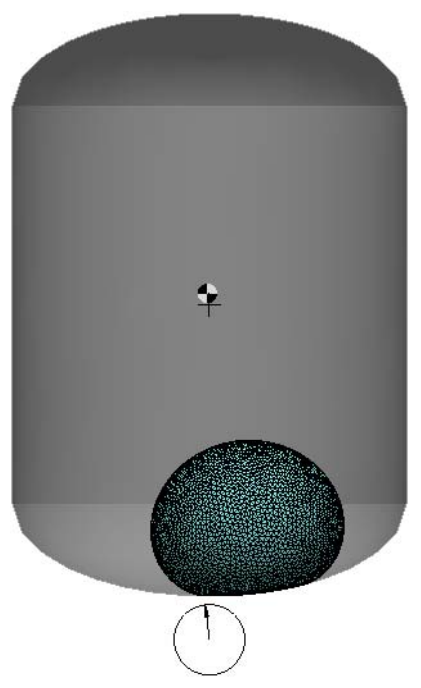

Case 4

Descent LOX

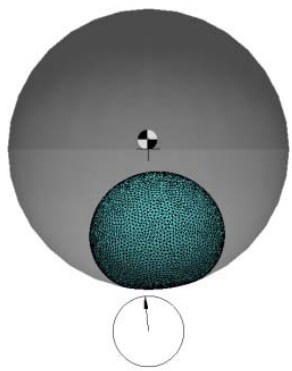

Case 5 Ascent LOX

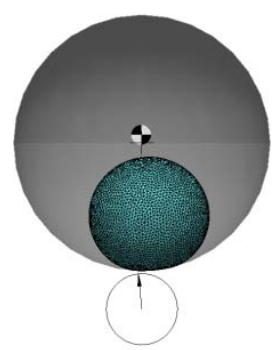

Case 6 Ascent LCH4

Figure 5. Ullage bubble shapes for the Altair vehicle tanks in LEO. The acceleration field is the result of a fixed Earth-spacecraft orientation plus orbital drag.

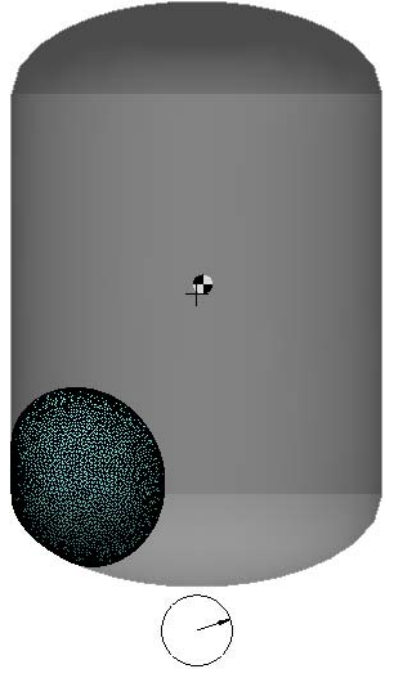

Case 11

Descent LH2

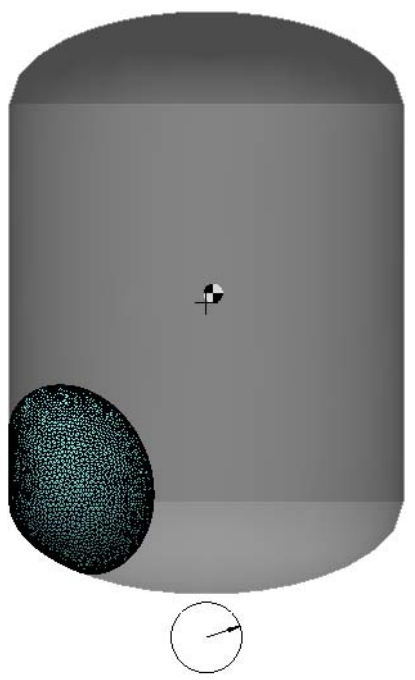

Case 12 Descent LOX

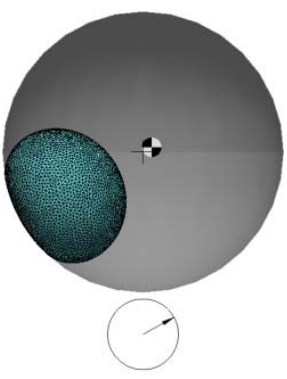

Case 13

Ascent LOX

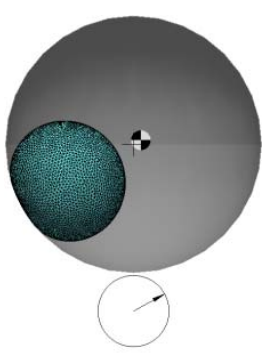

Case 14 Ascent LCH4

Figure 6. Ullage bubble shapes for the Altair vehicle tanks in LEO. The acceleration field is the result of a fixed Earth-spacecraft z-axis orientation plus a 20 minute period rotisserie-type rotation about the z-axis. 


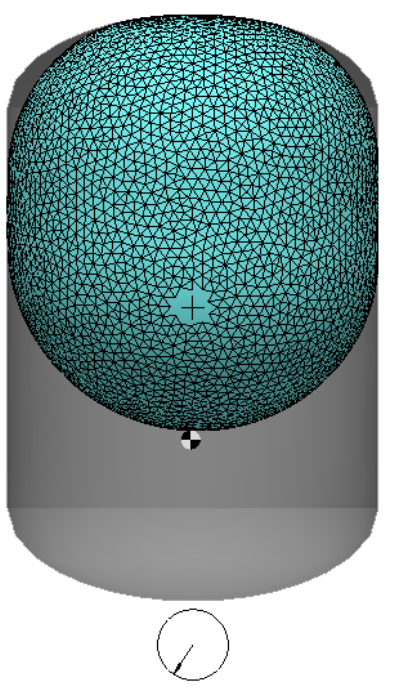

Case 7

Descent LH2

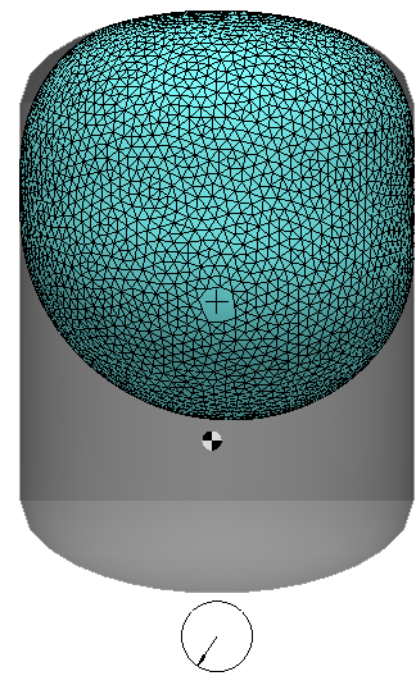

Case 8

Descent LOX

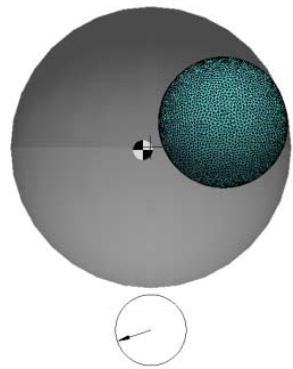

Case 9

Ascent LOX

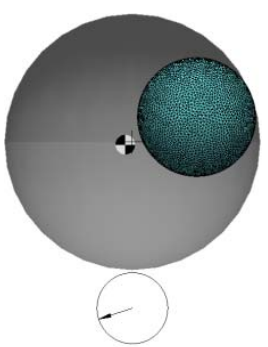

Case 10

Ascent LCH4

\section{Figure 7. Ullage bubble shapes for the Altair vehicle tanks in LLO. The acceleration field is the result of a} fixed Moon-spacecraft orientation.

\section{Summary}

The Surface Evolver code was used to model the equilibrium liquid-vapor interface configuration in exploration vehicle cryogenic propellant tanks in the low-gravity environments of LEO and LLO. A simple model of the EDS, Altair and CEV exploration vehicles was adopted from the ESAS report, and acceleration fields were calculated based on centrifugal and atmospheric drag forces. The resulting Bond numbers range from 0.02 to 14 , and show a variety of interesting fluid configurations in the tanks. Although the geometries investigated in this study are relatively simple, SE proves to be a unique and efficient algorithm to study equilibrium interface configurations in spacecraft propellant tanks. Not surprisingly, the ullage bubble can occur at the bottom (drain end) of the tank as a result of centrifugal forces, which could impact propellant management devices if they rely on liquid being available at that location. The results of this study, although specific to the spacecraft model adopted here, emphasize the utility of using SE to predict fluid configurations in low-gravity.

\section{Acknowledgements}

This work was supported by NASA through the Exploration Technology Development Program Office, Cryogenic Fluid Management project.

\section{References}

${ }^{1}$ NASA's Exploration Systems Architecture Study, Final Report, NASA-TM-2005-214062, 2005.

${ }^{2}$ Hastings, G. A., Hill, D. W., Satterlee, H. M., Seebold, J. G., NASA CR-65539, 1965.

${ }^{3}$ Brakke, K. A., Experimental Mathematics, Vol. 1, pp.141-165, 1992.

${ }^{4}$ Tegart, J., "Three-dimensional fluid interfaces in cylindrical containers," AIAA paper AIAA-91-2174, 27th Joint Propulsion Conference, Sacramento, CA, June 1991.

${ }^{5}$ Callahan, M., Concus, P., and Finn, R., "Energy minimizing capillary surfaces for exotic containers," Computing Optimal Geometries, AMS Selected Lectures in Mathematics. Amer. Math. Soc., 1991, pp.13-15.

${ }^{6}$ Mittelmann, H. D., "Symmetric capillary surfaces in a cube. Part 2: near the limit angle," Lect. Appl. Math., Vol. 29, 1993, pp.339-361.

${ }^{7}$ Ambrose, J., Yendler, B., and Collicott, S. H., "Modeling to Evaluate a Spacecraft Propellant Gauging System," Spacecraft and Rockets,Vol. 37, No. 6, 2000, pp.833-835.

${ }^{8}$ Collicott, S. H., "Convergence Behavior of Surface Evolver Applied to a Generic Propellant Management Device," J. Propulsion and Power, Vol. 17, No. 4, 2001, pp. 845-851

${ }^{9}$ Moe, K., and Moe, M. M., Planetary and Space Science, Vol.53, 2005, pp.793-801.

${ }^{10}$ Jacchia, L. G., Smithsonian Astrophysical Observatory Special Report 313, 1970; also available as NASA CR-112684. 
${ }^{11}$ Lemmon, E. W., Huber, M. L., and McLinden, M. O., "NIST Standard Reference Database 23: Reference Fluid Thermodynamic and Transport Properties-REFPROP," Version 8.0, National Institute of Standards and Technology, Standard Reference Data Program, Gaithersburg, 2007.

${ }^{12}$ Collicott, S. H., and Weislogel, M. M. "Computing Existence and Stability of Capillary Surfaces using Surface Evolver," AIAA Journal Vol. 42, No.2, 2004, pp.289-295.

${ }^{13}$ Chen, Y., Bacich, M. A., Nardin, C. L., Sitorus, A. T., Weislogel, M. M., "The Shape and Stability of Wall-Bound and Wall-Edge-Bound Drops and Bubbles,” Microgravity sci. technol., Vol.XVII, No.4, 2005, pp.14-24. 